\title{
NEMATODES ASSOCIATED WITH PLANT GROWTH INHIBITION IN THE WIELKOPOLSKA REGION
}

\author{
Grażyna Winiszewska ${ }^{1 *}$, Ewa Dmowska ${ }^{1}$, Aneta Chałańska ${ }^{2}$, Renata Dobosz ${ }^{3}$, Franciszek Kornobis ${ }^{1,7}$, \\ Krassimira Ilieva-Makulec ${ }^{4,8}$, Andrzej Skwiercz ${ }^{5}$, Stefan Wolny ${ }^{1,3}$, Elias Ishaqe ${ }^{6}$
}

\author{
${ }^{1}$ Museum and Institute of Zoology Polish Academy of Science, Wilcza 64, 00-679 Warszawa, Poland \\ ${ }^{2}$ Research Institute of Horticulture, Konstytucji 3 Maja 1/3, 96-100 Skierniewice, Poland \\ ${ }^{3}$ Institut of Plant Protection - National Research Institute, Władysława Węgorka 20, 60-318 Poznań, Poland \\ ${ }^{4}$ Centre of Ecological Research Polish Academy of Science, Konopnickiej 1, Dziekanów Leśny, 05-092 Łomianki, Poland \\ ${ }^{5}$ University of Warmia and Mazury, Prawocheńskiego 17, 10-722 Olsztyn, Poland \\ ${ }^{6}$ University of Al-Furat, DeirEzzor, Syria \\ ${ }^{7}$ Adam Mickiewicz University, Department of Animals Morphology, Faculty of Biology, Umultowska 89, 61-614 Poznań, Poland \\ ${ }^{8}$ Cardinal Stefan Wyszyński University, Institute of Ecology and Bioethics, Wóycickiego 1/3, 01-938 Warszawa, Poland
}

Received: May 15, 2012

Accepted: September 17, 2012

\begin{abstract}
The list of species of the plant parasitic nematodes presented in this paper (133 species belonging to 14 families) is based on the results of faunistic research conducted in the Wielkopolska region by Polish nematologists up until the year 2010, and the results obtained from the project "Elaboration of Innovative Methods for Rapid Identification of Nematodes Causing Damage to the Economy" managed by the Museum and Institute of Zoology of the Polish Academy of Sciences. During the two years of the project (2010-2011) we found 21 species of nematodes which had not yet been reported in the list of species from the Wielkopolska region. Two of them were reported for the first time in Poland.
\end{abstract}

Key words: plant parasitic nematodes, Wielkopolska, Poland

\section{INTRODUCTION}

The development of nematological research in the Wielkopolska region was influenced by the detection of the potato cyst nematode [Globodera rostochiensis (Wollenweber, 1923)] outbreaks. The potato cyst nematode is one of the most dangerous species of plant parasitic nematodes for agricultural production. Studies on this species were carried out mainly at the Institute of Plant Protection (IPP) in Poznan, created in 1951, now known as the Institute of Plant Protection - National Research Institute (IPP - NRI). With time, IPP research interests have expanded to other nematode species. Many years of research conducted by nematologists in the Wielkopolska region were focused on plant parasitic nematodes associated with agricultural crops (Wilski 1971; Radziwinowicz 1972; Wasilewska 1974; Kornobis 1983, 1993; Wolny 1986, 1989a, b, c, 1990; Kornobis and Ishaq 1990; Dobosz 1999), on weeds (Ishaq 1992; Kornobis and Wolny 1997; Dobosz et al. 2006), and planting trees and shrubs in forest nurseries (Wolny 1973, 1980; Skwiercz 2012). Data on plant-nematodes found in peat soils in Wielkopolska region has been published in the papers of Skwiercz (1989a, b).
Data on plant-nematodes occuring on tulip plantations are found in the publication of Chałańska and Skwiercz (2011). Information on the occurrence of species belonging to the Longidoridae, Xiphinematidae and Trichodoridae families are included in the papers of Szczygieł and Brzeski (1985) and Karnkowski (2005).

All these results were used as a comparative material for the research coordinated by the Museum and Institute of Zoology in the project "Elaboration of Innovative Methods for Rapid Identification of Nematodes Causing Damage to the Economy". For the project, nematodes were collected in a variety of environments: agriculture fields, forests, wooded areas, orchards, forest nurseries, ornamental plants plantations, and in mid-field shelterbelts that are so characteristic for Wielkopolska region.

\section{MATERIALS AND METHODS}

Research was carried out in the 2010-2011 time period on areas used for agricultural purposes, on ornamental plants in nurseries, orchards, forests, and wooded areas. 
Soil samples were collected in the rizosphere of the plants using a soil sampler (in diameter $30 \mathrm{~mm}$ ) to a depth of 30 $\mathrm{cm}$ (three puncture probes in an area of approximately 0.5 square meters). Nematodes were isolated from the soil using the Oostenbrink apparatus, modified Baermann method and centrifuge method. Cyst nematodes were extracted from the soil using a Fenwick can apparatus (Wilski 1967). Nematodes extracted from the soil were killed with hot water and preserved at liquid TAF [Tri-ethylamine, Aqua destillata, Formalin] (Wilski 1967). Nematodes identified based on morphological characteristics were classified according to the system adopted in the Fauna Europea (Bogdanowicz et al. 2008). Identified nematode species came from 110 samples.

\section{RESULTS AND DISCUSSION}

Analysis of data from the literature and the results from the project showed that in agricultural ecosystems and in natural environments of the Wielkopolska region, there are 133 species of herbivores and fungivores nematodes belonging to 14 families (Table 1). For most families, the number of species found in the Wielkopolska region was about half of the number of species previously reported from Poland (Fig. 1).

During the research period, two new species for Polish fauna were found: Aphelenchoides conimucronatus Bessarabova, 1966 and Bitylenchus parvus Allen, 1955.

Table 1. List of species of plant parasitic nematodes found in the Wielkopolska and the environment or place of the occurrence

\begin{tabular}{|c|c|}
\hline Species & Environment/place of occurrence \\
\hline 1 & 2 \\
\hline \multicolumn{2}{|r|}{ Longidoridae } \\
\hline L. attenuatus Hooper, 1961 & garden, hop field, meadow, orchad, woods \\
\hline L. elongatus (De Man, 1876) & $\begin{array}{l}\text { barley field, deciduous forest nursery, meadow, orchad, rape field, } \\
\text { shrubs in peat soils, weeds in cereal fields, woods }\end{array}$ \\
\hline L. euonymus Mali \& Hooper, 1973 & meadow, weeds in cereal fields, woods \\
\hline L. intermedius Kozłowska \& Seinhorst, 1979 & deciduous forest \\
\hline L. leptocephalus Hooper, 1961 & meadow \\
\hline Paralongidorus maximus (Bütschli, 1874) & garden, grapevine, woods \\
\hline \multicolumn{2}{|r|}{ Xiphinematidae } \\
\hline Xiphinema diversicaudatum (Micoletzky, 1927) & deciduous forest nursery, orchad, woods \\
\hline X. vuittenezi Luc, Lima, Weischer \& Flegg, 1964 & orchad \\
\hline Xiphinema sp. & fallow \\
\hline \multicolumn{2}{|r|}{ Trichodoridae } \\
\hline Paratrichodorus anemones (Loof, 1965) & mixed forest, meadow \\
\hline P. pachydermus (Seinhorst, 1954) & $\begin{array}{l}\text { barley field, beet field, coniferous forest nursery, deciduous forest } \\
\text { nursery, deciduous forest, field, grasses in peat soils, maize field, } \\
\text { meadow, mixed forest, orchad, rape field, rye field, shrubs in peat } \\
\text { soils, weeds in cereal fields, wheat field, woods }\end{array}$ \\
\hline P. teres (Hooper, 1962) & $\begin{array}{l}\text { barley field, coniferous forest nursery, deciduous forest, meadow, oat } \\
\text { field, orchad, ornamental deciduous nursery, rape, rye field, } \\
\text { weeds in cereal fields, wheat field }\end{array}$ \\
\hline Trichodorus cylindricus Hooper, 1962 & coniferous forest nursery \\
\hline T. primitivus (De Man, 1880) & $\begin{array}{l}\text { barley field, beet field, cereals, coniferous forest nursery, grasses in } \\
\text { peat soils, lupine field, maize field, oat field, orchad, rape field, shrubs } \\
\text { in peat soils, weeds in cereal fields, wheat field, woods }\end{array}$ \\
\hline T. similis Seinhorst, 1963 & coniferous forest nursery, grasses in peat soils, rape field, wheat field \\
\hline T. sparsus Szczygieł, 1968 & coniferous forest nursery \\
\hline T. variopapillatus Hooper, 1972 & grasses in peat soils, mixed forest \\
\hline T. viruliferus Hooper, 1963 & $\begin{array}{l}\text { barley field, coniferous forest nursery, deciduous forest nursery, } \\
\text { grasses in peat soils, lupine field, maize field, meadow, oat field, or- } \\
\text { chad, rape field, weeds in cereal fields, wheat field }\end{array}$ \\
\hline \multicolumn{2}{|r|}{ Aphelenchidae } \\
\hline Aphelenchus avenae Bastian, 1865 & $\begin{array}{l}\text { cereals, coniferous forest nursery, deciduous forest nursery, deciduous } \\
\text { forest, maize field, mixed forest,orchad, ornamental bulbs nursery, } \\
\text { ornamental conifer nursery, ornamental deciduous nursery, potatoe } \\
\text { field, weeds in cereal fields, woods }\end{array}$ \\
\hline A. eremitus Thorne, 1961 & coniferous forest nursery \\
\hline \multicolumn{2}{|r|}{ Aphelenchoididae } \\
\hline Aphelenchoides asterocaudatus Das, 1960 & triticale field \\
\hline A. bicaudatus (Imamura, 1931) & $\begin{array}{l}\text { coniferous forest nursery, deciduous forest nursery, field shelterbelt, } \\
\text { triticale field, weeds in cereal fields }\end{array}$ \\
\hline
\end{tabular}


$\frac{1}{\text { Aphelenchoides composticola Franklin, } 1957}$

A. conimucronatus Bessarabova, 1966

A. cyrtus Paesler, 1959

A. limberi Steiner, 1936

A. parietinus (Bastian, 1865)

A. saprophilus Franklin, 1957

Aphelenchoides sp.

Criconema annuliferum (De Man, 1921)

C. princeps (Andrássy, 1962)

C. sphagni Micoletzky, 1925

Criconemoides informis (Micoletzky, 1922)

C. mongolensis Andrássy, 1964

C. morgensis (Hofmänner, 1914)

C. parvus Raski, 1952

Mesocriconema curvatum (Raski, 1952)

M. pseudosolivagum (De Grisse, 1964)

M. rotundicaudatum (Loof, 1964)

M. rusticum (Micoletzky, 1915)

Mesocriconema solivagum (Andrássy, 1962)

Mesocriconema sphaerocephalum (Taylor, 1936)

Mesocriconema xenoplax (Raski, 1952)

Xenocriconemella macrodora (Taylor, 1936)

Hemicycliophora conida Thorne, 1955

H. thornei Goodey, 1963

H. typica De Man, 1921

Loofia thienemanni (Schneider, 1925)

\begin{tabular}{|c|c|}
\hline \multicolumn{2}{|c|}{ Paratylenchidae } \\
\hline Paratylenchus aciculus Brown, 1959 & rape field, wheat field \\
\hline P. bukowinensis Micoletzky, 1922 & $\begin{array}{l}\text { coniferous forest nursery, grasses in peat soils, maize field, potato } \\
\text { field, weeds in cereal fields }\end{array}$ \\
\hline P. microdorus Andrássy, 1959 & $\begin{array}{l}\text { beet field, coniferous forest nursery, grasses in peat soils, oat field, } \\
\text { rape field, weeds in cereal fields, wheat field }\end{array}$ \\
\hline P. nanus Cobb, 1923 & $\begin{array}{l}\text { coniferous forest nursery, grasses in peat soils, maize field, shrubs in } \\
\text { peat soils, weeds in cereal fields }\end{array}$ \\
\hline P. projectus Jenkins, 1956 & $\begin{array}{l}\text { barley field, beet field, coniferous forest nursery, deciduous forest } \\
\text { nursery, field shelterbelt, grasses in peat soils, maize field, meadow, } \\
\text { oat field, ornamental conifer nursery, rape field, shrubs in peat soils, } \\
\text { weeds in cereal fields, wheat field }\end{array}$ \\
\hline P. steineri Golden, 1961 & grasses in peat soils, weeds in cereal fields \\
\hline P. straeleni (De Coninck, 1931) & grasses in peat soils, shrubs in peat soils \\
\hline P. veruculatus $\mathrm{Wu}, 1962$ & coniferous forest nursery \\
\hline \multicolumn{2}{|c|}{ Telotylenchidae } \\
\hline Amplimerlinius globigerus Siddiqi, 1979 & beet field, grasses in peat soils, rye field \\
\hline A. macrurus (Goodey, 1932) & oat field \\
\hline Bitylenchus bryobius (Sturhan, 1966) & meadow, woods \\
\hline
\end{tabular}

coniferous forest nursery, deciduous forest nursery

maize field

triticale field

deciduous forest nursery

mixed forest

coniferous forest nursery, deciduous forest nursery

field shelterbelt

Criconematidae

beet field, coniferous forest nursery, grasses in peat soils, meadow, mixed forest, orchad, rape field, shrubs in peat soils, weeds in cereal fields, wheat field, woods

woods

grasses in peat soils

barley field, beet field, coniferous forest nursery, deciduous forest nursery, grasses in peat soils, lupine field, maize field, meadow, oat field, orchad, rape field, shrubs in peat soils, weeds in cereal fields, wheat field, woods

deciduous forest nursery

grasses in peat soils

weeds in cereal fields

barley field, beet field, deciduous forest nursery, lupine field, maize field, oat field, orchad, rape field, weeds in cereal fields, wheat field maize field

grasses in peat soils

beet field, grasses in peat soils, maize field, shrubs in peat soils, weeds in cereal fields

woods

maize field

barley field, grasses in peat soils, mixed forest, orchad, ornamental conifer nursery, ornamental deciduous nursery, rape field, shrubs in peat soils, woods

woods

Hemicycliophoridae

barley field, meadow, oat field, orchad, shrubs in peat soils, woods

shrubs in peat soils

shrubs in peat soils

Paratylenchidae

rape field, wheat field

field, weeds in cereal fields

beet field, coniferous forest nursery, grasses in peat soils, oat field, coniferous forest nursery, grasses in peat soils, maize field, shrubs in peat soils, weeds in cereal fields

barley field, beet field, coniferous forest nursery, deciduous forest weeds in cereal fields, wheat field

grasses in peat soils, weeds in cereal field 
Bitylenchus dubius (Bütschli, 1873)

B. parvus (Allen, 1955)

Geocenamus tenuidens Thorne \& Malek, 1968

Merlinius alboranensis (Tobar Jimenéz, 1970)

M. brevidens (Allen, 1955)

M. joctus (Thorne, 1949)

M. microdorus (Geraert, 1966)

M. nanus (Allen, 1955)

M. nothus (Allen, 1955)

Nagelus leptus (Allen, 1955)

N. obscurus (Allen, 1955)

N. camelliae (Kheiri, 1972)

N. microphasmis Loof, 1960

N. judithae (Andrássy, 1962)

N. lamelliferus (de Man, 1880)

Quinisulcius capitatus (Allen, 1955)

Sauertylenchus maximus (Allen, 1955)

S. lenorus (Brown, 1956)

S. quadrifer (Andrássy, 1954)

S. tartuensis (Krall, 1959)

S. tessellatus (Goodey, 1952)

S. tumensis Skwiercz, 1984

S. rugosus (Siddiqi, 1963) barley field, beet field, coniferous forest nursery, deciduous forest nursery, deciduous forest, field shelterbelt, grasses in peat soils, lupine field, maize field, meadow, oat field, orchad, ornamental conifer nursery, ornamental deciduous nursery,

ornamental plants, potato field, rape field, rye field, shrubs in peat soils, triticale field, weeds in cereal fields, wheat field, woods

woods

potato field, triticale field, weeds in cereal fields, wheat field,

barley field, beet field, woods

barley field, beet field, coniferous forest nursery, deciduous forest nursery, grasses in peat soils, lupine field, maize field, oat field, potato field, rape field, shrubs in peat soils, weeds in cereal fields, wheat field deciduous forest, maize field, meadow

barley field, beet field, coniferous forest nursery, deciduous forest nursery, deciduous forest, field shelterbelt, grasses in peat soils, lupine field, maize field, meadow, oat field, orchad, potato field, rape field, weeds in cereal fields, wheat field, woods

barley field, beet field, coniferous forest nursery, deciduous forest nursery, deciduous forest, lupine field, maize field, meadow, oat field, potato field, rape field, rye field, weeds in cereal fields, woods

barley field, beet field, deciduous forest, grasses in peat soils, lupine field, maize field, meadow, oat field, rape field, weeds in cereal fields, wheat field, woods

shrubs in peat soils

barley field, grasses in peat soils, oat field, weeds in cereal fields, wheat field

weeds in cereal fields

barley field, beet field, coniferous forest nursery, deciduous forest nursery, lupine field, maize field, meadow, mixed forest, oat field, rape field, wheat field, woods

beet field, rape field, weeds in cereal fields, woods

grasses in peat soils

maize field

barley field, beet field, coniferous forest nursery, field shelterbelt, grasses in peat soils, lupine field, meadow, oat field, orchad, ornamental conifer nursery, rape field, shrubs in peat soils, weeds in cereal fields, wheat field, woods

barley field, beet field, deciduous forest

barley field, coniferous forest nursery, grasses in peat soils, maize field, rape field, wheat field

barley field, beet field, deciduous forest nursery, grasses in peat soils, lupine field, maize field, meadow, mixed forest, oat field, orchad, rape field, weeds in cereal fields, wheat field

coniferous forest nursery, grasses in peat soils, maize field, oat field, weeds in cereal fields, wheat field

grasses in peat soils

coniferous forest nursery

Heteroderidae

Globodera artemisiae (Eroshenko \& Kazachenko, 1972)

G. rostochiensis (Wollenweber, 1923)

weeds in cereal fields

barley field, beet field, coniferous forest nursery, oat field, rape field, weeds in cereal fields, wheat field

barley field, beet field, lupine field, maize field, oat field, rape field, weeds in cereal fields, wheat field

weeds in cereal fields

lupine field

rape field, weeds in cereal fields

black fallow

weeds in cereal fields, wheat field 
Heterodera humuli Filipjev, 1934

H. schachtii Schmidt, 1871

H. trifolii Goffart, 1932

Heterodera sp.

Punctodera punctata (Thorne, 1928)

Helicotylenchus canadensis Waseem, 1961

H. digonicus Perry, 1959

H. exallus Sher, 1966

H. pseudorobustus (Steiner, 1914)

H. varicaudatus Yuen, 1964

H. vulgaris Yuen, 1964

H. pseudodigonicus Szczygieł, 1970

Rotylenchus agnetis Szczygieł, 1968

R. buxophilus Golden, 1956

R. goodeyi Loof \& Oostenbrink, 1958

R. quartus (Andrássy, 1958)

R. robustus (De Man, 1876)

Rotylenchus sp.

Meloidogyne hapla Chitwood, 1949

Meloidogyne sp.

Hirschmanniella gracilis (De Man, 1880)

Pratylenchoides crenicauda Winslow, 1958

P. laticauda Braun \& Loof, 1967

Pratylenchus crenatus Loof, 1960

P. fallax Seinhorst, 1968

P. flakkensis Seinhorst, 1968

P. neglectus (Rensch, 1924)

P. penetrans (Cobb, 1917)

P. pinguicaudatus Corbett, 1969

P. pratensis (De Man, 1880)

P. thornei Sher \& Allen, 1953

Pratylenchus sp. hop field, weeds in cereal fields

barley field, beet field, lupine field, maize field, meadow, oat field, rape field, wheat field

barley field, beet field, lupine field, rape field, weeds in cereal fields, wheat field

deciduous forest, maize field, meadow

barley field, weeds in cereal fields

Hoplolaimidae

barley field, beet field, cereals, coniferous forest nursery, garlic field, grasses in peat soils, oat field, rape field, weeds in cereal fields, wheat field

barley field, beet field, coniferous forest nursery, deciduous forest nursery, garlic field, grasses in peat soils, oat field, ornamental conifer nursery, rape field, weeds in cereal fields, wheat field, woods

grasses in peat soils

barley field, beet field, coniferous forest nursery, deciduous forest nursery, deciduous forest, grasses in peat soils, lupine field, maize field, meadow, mixed forest, oat field, ornamental conifer nursery, rape field, shrubs in peat soils, weeds in cereal fields, wheat field, woods garlic field, potato field, woods

beet field

maize field

barley field, ornamental deciduous nursery, woods

coniferous forest nursery, woods

barley field, beet field, coniferous forest nursery, grasses in peat soils, maize field, mixed forest, rape field, weeds in cereal fields, woods coniferous forest nursery, deciduous forest nursery, grasses in peat soils, woods

beet field, coniferous forest nursery, deciduous forest nursery, grasses in peat soils, rape field, weeds in cereal fields, woods woods

Meloidogynidae

coniferous forest nursery, barley field, weeds on meadow, weeds in cereal fields,

potato field, rye field

Pratylenchidae

grasses in peat soils

coniferous forest nursery, weeds on meadow

shrubs in peat soils

barley field, beet field, coniferous forest nursery, deciduous forest nursery, grasses in peat soils, las mixed forest, lupine field, maize field, meadow, oat field, potato field, rape field, shrubs in peat soils, weeds in cereal fields, wheat field, woods

coniferous forest nursery, deciduous forest nursery, grasses in peat soils, maize field, oat field, potato field, rape field, shrubs in peat soils barley field, deciduous forest nursery, grasses in peat soils, lupine field, oat field, rape field, shrubs in peat soils, weeds in cereal fields barley field, beet field, deciduous forest nursery, field shelterbelt, grasses in peat soils, lupine field, maize field, meadow, meadow, oat field, orchad, ornamental conifer nursery, potato field, rape field, shrubs in peat soils, weeds in cereal fields, wheat field

barley field, coniferous forest nursery, deciduous forest nursery, grasses in peat soils, maize field, oat field, orchad, potato field, rape field, shrubs in peat soils, weeds in cereal fields, wheat field weeds in cereal fields deciduous forest nursery, weeds in cereal fields beet field, maize field, oat field, rape field, weeds in cereal fields, wheat field

field shelterbelt, maize field, meadow, ornamental deciduous nursery, strawberry field, woods 


\begin{tabular}{|c|c|}
\hline 1 & 2 \\
\hline & Anguinidae \\
\hline Ditylenchus clarus Thorne \& Malek, 1968 & weeds in cereal fields \\
\hline D. convallariae Sturhan \& Friedman, 1965 & coniferous forest nursery \\
\hline D. destructor Thorne, 1945 & beet field, potato field, rape field, weeds in cereal fields \\
\hline D. dipsaci (Kühn, 1857) & $\begin{array}{l}\text { barley field, oat field, potato field, rape field, rye field, weeds in cereal } \\
\text { fields, wheat field }\end{array}$ \\
\hline D. equalis Heyns, 1964 & beet field, rape field, weeds in cereal fields \\
\hline D. exilis Brzeski, 1984 & beet field, weeds in cereal fields \\
\hline D. medicaginis Wasilewska, 1965 & $\begin{array}{l}\text { barley field, beet field, coniferous forest nursery, lupine field, oat field, } \\
\text { rape field, weeds in cereal fields }\end{array}$ \\
\hline $\begin{array}{l}\text { D. myceliophagus Goodey, } 1958 \\
\text { D. intermedius (de Man, 1880) }\end{array}$ & $\begin{array}{l}\text { deciduous forest nursery, potato field } \\
\text { deciduous forest nursery, potato field }\end{array}$ \\
\hline Nothotylenchus ferepolitor Kazachenko, 1980 & beet field \\
\hline Safianema anchilisposomus (Tarjan, 1958) & deciduous forest nursery \\
\hline
\end{tabular}

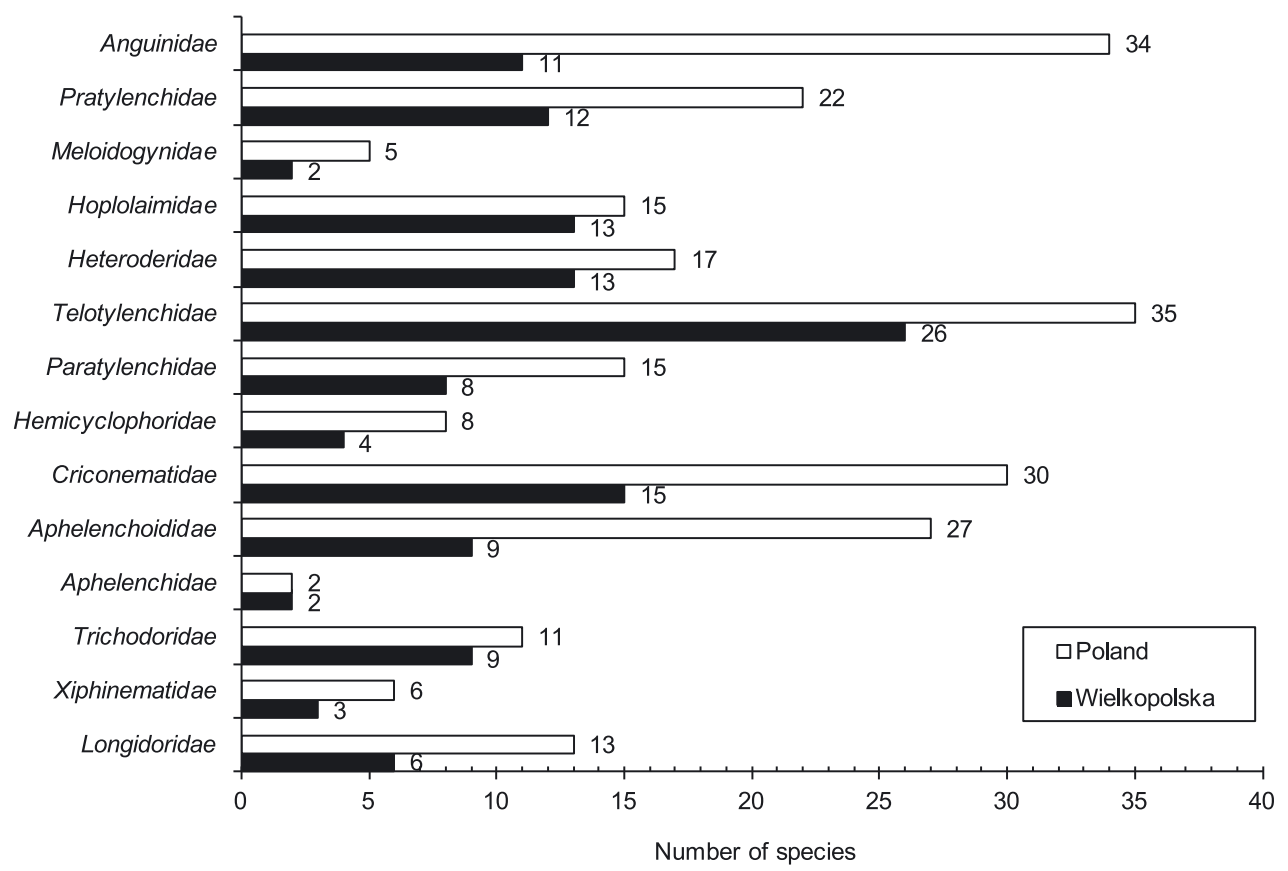

Fig. 1. Comparison of the number of species of plant parasitic nematodes from some families reported from Poland and found in Wielkopolska

The species $A$. conimucronatus was previously reported in Russia, Ukraine, Czech Republic (http:// www. faunaeur.org), and Slovakia (Hánĕl and Čerevková 2010). Up till now, Bitylenchus parvus was recognized in the soil around the roots of plants cultivated in the area of Cyprus, Hungary and Italy (http://www.faunaeur. org). Moreover, 16 species which had not been previously reported in Wielkopolska were found: Longidorus euonymus Mali \& Hooper, 1973, L. intermedius Kozłowska \& Seinhorst, 1979, L. leptocephalus Hooper, 1961 and Paralongidorus maximus (Bütschli, 1874) belonging to the Longidoridae family; Xiphinema vuittenezi Luc, Lima, Weischer \& Flegg, 1964 belonging to the Xiphinematidae family; Paratrichodorus anemones (Loof, 1965) belonging to the Trichodoridae family; Aphelenchoides cyrtus Paesler, 1959, A. parietinus (Bastian, 1865) and Aphelenchoides sp. belonging to the Aphelenchoididae family; Criconema princeps (Andrássy, 1962), Mesocriconema solivagum (Andrássy, 1962) and Xenocriconemella macrodora (Taylor, 1936) belonging to the Criconematidae family, Bitylenchus bryobi- us (Sturhan, 1966), Merlinius alboranensis (Tobar Jimenéz, 1970) and Scutylenchus lenorus (Brown, 1956) belonging to the Telotylenchidae family; and Helicotylenchus varicaudatus Yuen, 1964 belonging to the Hoplolaimidae family.

Specimens identified as Rotylenchus sp. and Pratylenchus sp. are probably new species, what will be finally confirmed by molecular tests.

\section{CONCLUSIONS}

Due to two-year studies carried out in the project "Elaboration of Innovative Methods for Rapid Identification of Nematodes that Cause Damage to the Economy", which involved a wide range of environments, the list of 112 species recorded in the Wielkopolska region up till 2010 is extended by further 21 species, including 2 species new for polish fauna: A. conimucronatus and B. parvus. 


\section{REFERENCES}

Bogdanowicz W., Chudzicka E., Pilipiuk I., Skibińska E. (eds). 2008. Fauna Polski. Muzeum i Instytut Zoologii PAN, Warszawa 3, 603 pp.

Chałańska A., Skwiercz A. 2011. Parasitic nematodes on Polish tulip plantations. J. Plant Prot. Res. 51 (1): 66-71.

Dobosz R. 1999. Additional data on plant parasitic nematodes on sugarbeet in the Wielkopolska region in Poland. J. Plant Prot. Res. 39 (2): 107-108.

Dobosz R., Obrępalska-Stęplowska A., Kornobis S. 2006. Globodera artemisiae (Eroshenko et Kazachenko, 1972) (Nematoda: Heteroderidae) from Poland. J. Plant Prot. Res. 46 (4): 403-407.

Háněl L., Čerevková A. 2010. Prvé nálezy pôdnych nematód (Nematoda) pre faunu Slovenska z CHKO Vihorlat. Folia Faunistica Slovaca 15 (10): 95-98.

Ishaqe E. 1992. Plant-parasitic nematodes associated with weeds in spring cereal fields in the region of Wielkopolska. Rocz. Nauk Rol. Seria E - Ochrona Roślin 22 (1/2): 7-30.

Karnkowski W. 2005. Ocena występowania długaczy (Longidorus spp.) i sztylaków (Xiphinema spp.) (Nematoda: Longidoridae) na podstawie badań gleby przeprowadzonych w centralnym laboratorium głównego inspektoratu ochrony roślin i nasiennictwa. [Evaluation of occurrence of needles nematodes (Longidorus spp.) and dagger nematodes (Xiphinema spp.)(Nematoda: Longidoridae) on the basis of soil examination performed in the Central Laboratory of the Main Inspectorate of Plant Protection and Seed Inspection]. Prog. Plant Prot./Post. Ochr. Roślin 45 (2): 764-767.

Kornobis S. 1983. Materiały do znajomości nicieni występujących w uprawach kukurydzy w Wielkopolsce. [Plant parasitic nematodes associated with poor growth of maize in Wielkopolska]. Z. Probl. Post. Nauk Roln. 278: 139-148.

Kornobis S. 1993. Plant parasitic nematodes associated with poor growth of lupines in the Wielkopolska region. Rocz. Nauk Rol. Seria E - Ochrona Roślin 23 (1/2): 93-95.

Kornobis S., Ishaqe E. 1990. Materiały do znajomości nicieni stowarzyszonych z objawami zahamowania wzrostu roślin buraka cukrowego w Wielopolsce. [Plant parasitic nematodes associated with poor growth of sugar beet in the Wielkopolska region]. Prace Nauk. Inst. Ochr. Roślin 32 (1/2): 93-98.

Kornobis S., Wolny S. 1997. Occurence of plant parasitic nematodes on weeds in agrobiocenosis in the Wielkopolska region in Poland. Fundam. Appl. Nematol. 20 (6): 627-632.

Radziwinowicz J. 1972. Badania nad występowaniem nicieni szkodników roślin na ziemniakach w polu i przechowalniach. [Investigations on the occurrence of plant parasitic nematodes on potatoes in fields and clamps]. Prace Nauk. Inst. Ochr. Roślin 14 (1): 157-168.

Skwiercz A.T. 1989a. Plant parasitic nematodes in the peat soils in Poland, Part I. Biocenotic analyse. Rocz. Nauk Rol. Seria E - Ochrona Roślin 19 (1/2): 91-99.
Skwiercz A.T. 1989b. Plant parasitic nematodes in the peat soils in Poland, Part II. Frequency of occurrence and population density in different chemical properties of peat. Rocz. Nauk Rol. Seria E - Ochrona Roślin 19 (1/2): 101-111.

Skwiercz A.T. 2012. Nematodes (Nematoda) in Polish forests. I. Species inhabiting soils of nurseries. J. Plant Prot. Res. 52 (1): 169-179.

Szczygieł A., Brzeski M.W. 1985. Atlas of Plant Parasitic Nematodes of Poland. Distribution of Longidoridae, Xiphinemidae and Trichodoridae. Eur. Plant Parasitic Nematode Survey, $32 \mathrm{pp}$.

Wasilewska L. 1974. Number, biomas and metabolic activity of nematodes of two cultivated fields in Turew. Z. Probl. Post. Nauk Roln. 154: 419-442.

Wilski A. 1967. Nicienie Szkodniki Roślin Uprawnych. PWRiL, Warszawa, $336 \mathrm{pp}$.

Wilski A. 1971. Występowanie nicieni z rodzaju Heterodera w glebach województwa poznańskiego. [The occurrence of Heterodera cysts in soils of the province Poznan]. Prace Nauk. Inst. Ochr. Roślin 13 (1): 195-200.

Wolny S. 1973. Przyczynek do poznania fauny nicieni szkółek sosnowych Leśnego Zakładu Doświadczalnego Siemianice, powiat Kępno. [A contribution to the knowledge of the nematode fauna in pine seed-beds of the forest experimental farm, Siemianice, district Kepno]. Prace Nauk. Inst. Ochr. Roślin 15 (2): 127-132.

Wolny S. 1980. Nicienie, pasożyty roślin w szkółkach zadrzewieniowych. [Plant parasitic nematodes in tree nurseries]. Z. Probl. Post. Nauk Rol. 232: 121-132.

Wolny S. 1986. Występowanie nicieni - pasożytów roślin wyższych w uprawach zbóż jarych na terenie Wielkopolski. Mat. 26. Sesji Nauk. Inst. Ochr. Roślin, Cz. II: 45-46.

Wolny S. 1989a. Nicienie - pasożyty roślin wyższych stowarzyszone z objawami zahamowania wzrostu roślin jęczmienia jarego w Wielkopolsce. [Plant parasitic nematodes associated with poor growth of spring barley in the Wielkopolska region]. Prace Nauk. Inst. Ochr. Roślin 31 (1): 5-16.

Wolny S. 1989b. Nicienie - pasożyty roślin wyższych stowarzyszone $\mathrm{z}$ objawami zahamowania wzrostu roślin pszenicy jarej w Wielkopolsce. [Plant parasitic nematodes associated with poor growth of spring wheat in the Wielkopolska region]. Prace Nauk. Inst. Ochr. Roślin 31 (1): 17-28.

Wolny S. 1989c. Nicienie - pasożyty roślin wyższych stowarzyszone z objawami zahamowania wzrostu roślin owsa $\mathrm{w}$ Wielkopolsce. [Plant parasitic nematodes associated with poor growth of oat in the Wielkopolska region]. Prace Nauk. Inst. Ochr. Roślin 31 (1): 29-40.

Wolny S. 1990. Materiały do znajomości nicieni - pasożytów roślin wyższych stowarzyszonych z objawami zahamowania wzrostu roślin rzepaku ozimego w Wielkopolsce. [Plant parasitic nematodes associated with poor growth of winter rape in the Wielkopolska region ]. Prace Nauk. Inst. Ochr. Roślin 32 (1): 85-91. 